2008

\title{
A New System to Acquire and Restore Document Shape and Content
}

George V. Landon

Cedarville University, georgelandon@cedarville.edu

W. Brent Seales

Duncan Clarke

Follow this and additional works at: https:// digitalcommons.cedarville.edu/ engineering_and_computer_science_publications

Part of the Computer Engineering Commons

\section{Recommended Citation}

Landon, George V.; Seales, W. Brent; and Clarke, Duncan, "A New System to Acquire and Restore Document Shape and Content" (2008). Engineering and Computer Science Faculty Publications. 404.

https://digitalcommons.cedarville.edu/engineering_and_computer_science_publications/404 


\section{A New System to Acquire and Restore Document Shape and Content}

\author{
George V. Landon \\ Eastern Kentucky University \\ Richmond, KY 40475
}

\author{
W. Brent Seales \\ University of Kentucky \\ Lexington, KY 40506-0495
}

\author{
Duncan Clarke \\ Fremont Associates, LLC \\ Camden, SC 29020-4316
}

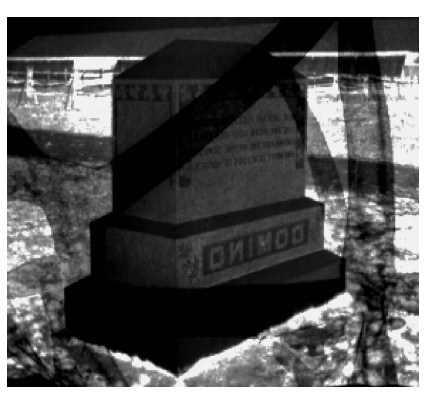

(a) The imaged back-lit film.

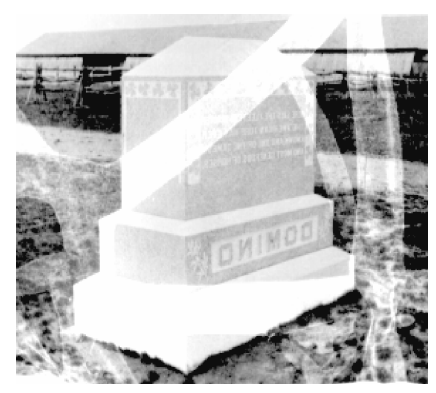

(b) The inverted negative.

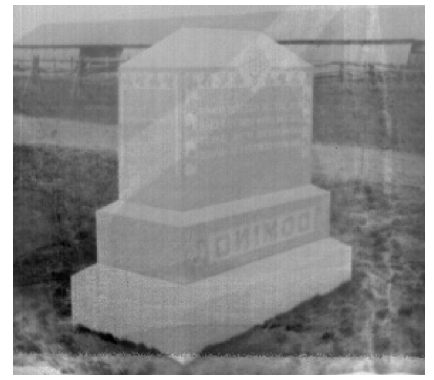

(c) The photometrically corrected image (variance map).

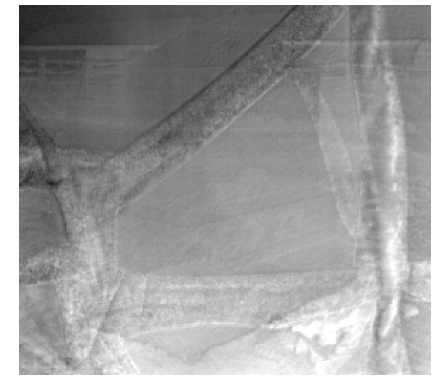

(d) The estimated surface orientations (distortion map).

Figure 1: A scan of the negative shown in Fig. 2: An example photographic recording of a tombstone.

\begin{abstract}
We have developed a system that can acquire and restore not only a distorted single-page document, but also more complicated multilayer film negatives. In this work, we demonstrate a new imagebased document acquisition system, based on single-scatter diffuse transmission, that captures the intrinsic intensity information and shape distortion of documents. Using a simple to build and costeffective camera and LCD system, the data is acquired to allow for automatic photometric and geometric correction of most documents. We show the results of our system with single sheet nonplanar documents. Furthermore, to show the robustness of this system, we demonstrate the restoration on safety acetate film negatives over 70 years old that have been damaged due to shrinkage and other forms of deterioration.
\end{abstract}

Keywords: diffuse transmission, rear-illumination document scanning, document restoration, shape acquisition, transparency, acetate negative

\section{Introduction}

Much of the current research in the area of document imaging has focused in document acquisition and restoration and, in particular, digitizing bound books or manuscript pages. Other documents, such as heavily damaged single-page documents or deteriorated photographic negatives, have typically been overlooked. While

Copyright $(\subset 2008$ by the Association for Computing Machinery, Inc.

Permission to make digital or hard copies of part or all of this work for personal or classroom use is granted without fee provided that copies are not made or distributed for commercial advantage and that copies bear this notice and the full citation on the first page. Copyrights for components of this work owned by others than ACM must be honored. Abstracting with credit is permitted. To copy otherwise, to republish, to post on servers, or to redistribute to lists, requires prior specific permission and/or a fee. Request permissions from Permissions Dept, ACM Inc., fax +1 (212) 869-0481 or e-mail permissions@acm.org.

PROCAMS 2008, Marina del Rey, California, August 10, 2008

(c) 2008 ACM 978-1-60558-272-6/08/0008 $\$ 5.00$ these types of documents are only a subset of all documents, many of the items in these collections contain subjects of extreme historical significance. Therefore, digitally preserving and restoring these deteriorating documents is an urgent challenge that requires an easily-accessible solution.

For instance, photographic negatives, taken from 1925 to as late as 1955, are suffering devastating forms of deterioration [Horvath 1987]. These three decades encompass vast and diverse collections of safety negatives. Safety negatives were developed to move away from the ammability of cellulose nitrate, which was used in still photography until the early 1920 s. The safety film that emerged was varied in its composition but largely based on cellulose diacetate. This new material lessened the risk of fire damage, but was not an ideal film base because of its tendency to absorb moisture causing dimensional deformations. In fact, it has been discovered that the chemical decomposition of this acetate, or base, layer, even under proper conservation, causes profound distortion in the film content as shown in Fig. 2(a) 2(b). When imaged, the effects of these distortions are obvious (see Fig. 1(a)).

The chemical deterioration of the acetate can only be slowed, not stopped [Horvath 1987]. However, the only currently accepted solution to restoring these negative is destructive physical separation of the layers [Chicago Albumen Works 2008]. The size and importance of the affected photographic collections cannot be overstated, with many individual collections containing over 100,000 negatives. The reality of budgeting, space constraints, and personnel limitations has led to a situation where damage is continuing and placed many important items at risk of complete loss. This has created an urgent need for a technique that can capture the information in each of the negatives of a large collection before the damage causes a complete and irretrievable loss of information.

Acquisition and restoration of general document types has been given focus by many groups who have made a great deal of progress in creating fast and accurate digitization systems. Currently, restoration of standard documents typically consists of correcting geometric and photometric distortions. Some works have focused mainly on geometric correction of distorted documents [Zhang et al. 2007] [Brown and Seales 2004]. Other projects have fo- 


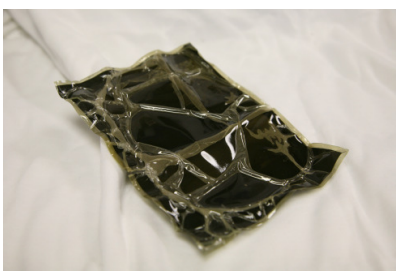

(a) Base layer

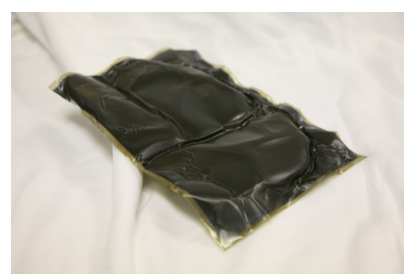

(b) Emulsion layer
Figure 2: A deteriorated safety acetate film negative.

cused more on photometric correction of documents [Landon et al. 2007] [Sun et al. 2005]. While others have relied on assumed document shapes to provide photometric and geometric corrections for objects such as bound books [Cao et al. 2003] and folded documents [Brown and Tsoi 2006]. Research has also been performed to scan documents that are not typically visible with normal imaging devices [Seales and Lin 2004].

In addition, a significant contribution of work has focused on the restoration of deteriorated photographs. Digital Inpainting [Bertalmio et al. 2000] provides an efficient procedure for restoring areas of loss in digital images. Inpainting has been improved in many ways since [Bertalmio et al. 2003] [Criminisi et al. 2004] [Roth and Black 2005], however, these procedures assume total loss of data in areas requiring restoration. The topic of damaged photograph restoration has been addressed specifically with image and signal processing. Content-based representation was used to assist in automatic and semi-automatic restorations [Ardizzone et al. 2006]. Reflective light imaging has also been used to detect blotches that have not fully destroyed the underlying content [Bruni et al. 2007]. Once detected, the content is extracted from the blotches to remove the deterioration. In a slightly different direction, a technique was developed to remove reflections from within the photographic content itself [Szeliski et al. 2000]. For an overview of of photograph restoration techniques, the reader may refer to [Stanco et al. 2003]. However, these methods generally focus on a scanned image of a photograph and usually only handle standard photographic prints. One project that does work directly with glass plate negatives [Stanco et al. 2004] uses rigid transformations to assemble broken photographs.

While these works have provided a great deal of progress toward acquiring many types of documents, they do not provide a way to capture documents with transparent properties. There has been work to acquire shape and optical properties of general transparent objects. One technique makes assumptions about the object shape to reconstruct the surface [Ben-Ezra and Nayar 2003]. In contrast, a new method using scatter tracing [Morris and Kutulakos 2007] acquires the outermost surface of complex transparent objects using assumptions about the object composition. For some applications, recording only the light transport of a scene is required. Specifically, environment matting [Zongker et al. 1999] [Chuang et al. 2000] uses a novel method for capturing the light transport through a scene.

In this work, we present a system that can acquire and restore not only a distorted single-page document, but also complicated multi-layer film negatives. This new image-based document acquisition system segments the content of the document from the surface shape to capture the intrinsic intensity information and shape distortion as independent variables in the observed intensity. This is done by assembling a cost-effective scanning system composed of a LCD as an active light-table, digital video camera, and a laptop computer. The presented method takes advantage of single-scatter diffusion to approximate shape and intensity information of a document which allows for automatic photometric and geometric correction of most documents.

This technique will be demonstrated with an acquisition method on two different document types. First, we show the results of our system with single-layer non-planar documents such as a single sheet of plain paper with text on one or both sides. Next, we show an application of this scanner that provides the first known attempt at providing a solution for digital restoration of deteriorated photographic negatives. The multiple layers of these films are continually separating which causes distortions both photometrically and geometrically when they are imaged. The system described here provides a virtual restoration without any physical manipulation of the negatives.

\section{Image-Based Document Scanner}

The basis of this work takes advantage of the isotropic transmissivity of most document substrates. Presented in this section is a method to isolate the intrinsic opacity and surface orientation from each observed pixel in the imaging device. By decoupling the surface shape from the document content, direct methods are available to restore distortions caused by imaging damaged documents.

The design of this scanner relies on the premise that all necessary information in a document can be obtained with rear-illumination. While there are some documents where this assumption fails, such as totally opaque materials, the vast majority of documents have some form of transmission.

\subsection{Document Transmissivity}

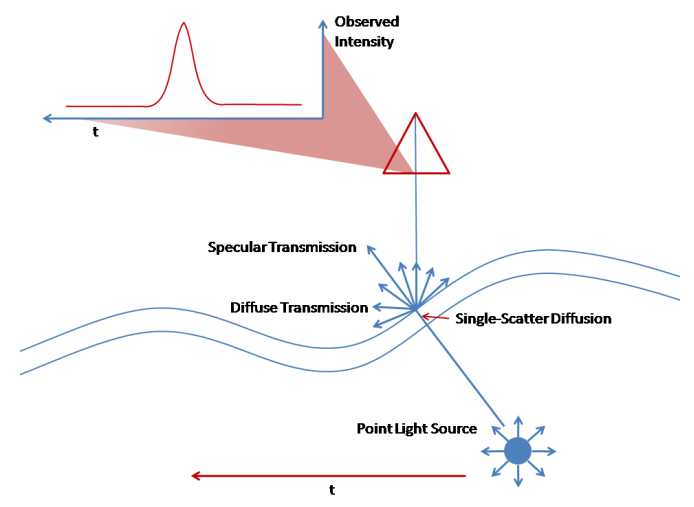

Figure 3: Diffuse single-scatter transmission of a back-lit light source.

In the case of modern paper, chemical processes are used to render wood pulp to the necessary composition with the correct balance of rigidity and transmissivity delivering a highly isotropic scattering of transmitted light. Paper can be accurately modeled as a diffuse reflector [Horn 1970] and consequently a diffuse transmitter of light. With a similar result, photographic negatives contain an emulsion layer composed of densely packed silver halide crystals that create diffuse transmission.

For a single-layer document, the diffuse transmission of light can be approximated as a single-scatter diffusion. This singlescattering can be approximated for diffuse transmission [Chandrasekhar 1960] as:

$$
I=I_{0} e^{\frac{-\tau}{\mu}}+\frac{1}{4 \pi} \omega_{0} I_{0} \frac{\mu_{0}}{\mu+\mu_{0}}\left(e^{\frac{-\tau_{1}}{\mu}}-e^{\frac{-\tau_{1}}{\mu_{0}}}\right)
$$


where $\mu_{0}$ is the cosine of the angle between the incident light and surface normal, $\mu$ is the cosine of the angle between the outgoing light and surface normal, $\omega_{0}$ is the phase function, $I_{0}$ is the incoming intensity, and $\tau_{1}$ is the material thickness.

The single-scatter transmission has been well studied in the area of computer graphics. Chandrasekhar's work has been used to create an efficient rendering system for thin semi-transparent objects [Frisvad et al. 2005]. Moreover, the area of plant/leaf rendering has been thoroughly studied [Wang et al. 2006] [Govaerts et al. 1996] [Ganapol et al. 1998] with respect to single-scatter transmission.

Fig. 3 shows a particular case where a light source is being translated approximately parallel to semi-planar object. For a singlepixel observation, as the light translates, the intensity follows a cosine-like response. Where the incident light, $l_{0}$, is approximately parallel to the surface normal, $n$, the cosine of this angle can be calculated as $\mu_{0}=l_{0} \cdot-n$. Furthermore, in the case of diffuse transmission, an assumption can be made that the greatest transmitted intensity will occur when $l_{0} \simeq n$. Therefore, for the purpose of this work, we will assume to be working in the case when $\mu_{0}$ is approximately 1 .

For diffuse materials, especially paper, it is safe to model the material as a translucent material with a highly diffuse transmission of light. Therefore, the phase function, can be modeled with isotropic scattering, thus $\omega_{0}$ becomes a constant 1 . The direct transmission can be safely ignored for highly diffuse materials, so singlescattering becomes the only factor in light transmission through the material. Considering these assumptions, Eq. 1, can be approximated as:

$$
I=\frac{1}{4 \pi} I_{0} \frac{1}{\mu+1}\left(e^{\frac{-\tau_{1}}{\mu}}-e^{-\tau_{1}}\right)
$$

where $\mu$ is the only varying quantity across the surface while $\tau_{1}$ and $I_{0}$ stay constant.

However, for documents that are mostly specular transmissive, surface normal no longer plays a large role in the intensity of transmitted light. Therefore, the following scanning process only works well for documents that exhibit diffuse transmission.

For photographic negatives, the two most important components to be considered are the emulsion layer and base layer. The emulsion layer contains the photographic content of the film, while the base layer provides support and rigidity to the film. Therefore, the base layer itself contains no relevant information but only provides the physical stability necessary to keep the emulsion intact.

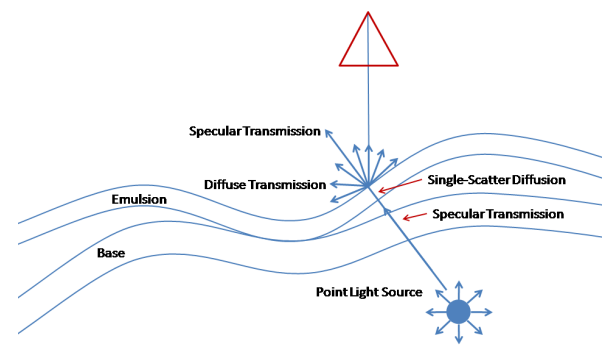

Figure 4: The transmission of light through a deteriorated photographic negative.

For this work, we found that it is safe to ignore the reflective and refractive properties of the acetate layer and to only focus on direct, or specular, transmission as shown in Fig. 4.

\subsection{The Scanning Process}

To obtain the transmissive properties of a document, a two-pass scanning process is performed. Using a LCD as an orthographic projector, time-evolving Gaussian stripe is displayed as the backprojected calibration pattern. We display two stripes, a vertical Gaussian stripe given by

$$
G\left(u ; u_{t}, \sigma_{u}\right)=d e^{\frac{-\left(u-u_{t}\right)^{2}}{2 \sigma_{u}^{2}}}
$$

and a horizontal Gaussian stripe given by

$$
G\left(v ; v_{t}, \sigma_{v}\right)=d e^{\frac{-\left(v-v_{t}\right)^{2}}{2 \sigma_{v}^{2}}}
$$

where $u$ and $v$ are display coordinates, $u_{t}$ and $v_{t}$ are the current positions of the stripe, $\sigma_{u}$ and $\sigma_{v}$ are the Gaussian variance, and $d$ represents the maximum intensity value available in the display.

The initial pass is captured with only the LCD in the scene and is only performed once if the relative poses remain static between the display and camera. This acquires the base calibration for each camera pixel giving the 2D Gaussian parameters $\sigma_{x}, \sigma_{y}, x_{0}$, and $y_{0}$ in camera coordinates. By capturing this initial pass, the scanning process is held in a completely image-based domain without the need for metric calibration. Consequently, there is no calculated correlation between the displayed Gaussian parameters and their imaged equivalents.

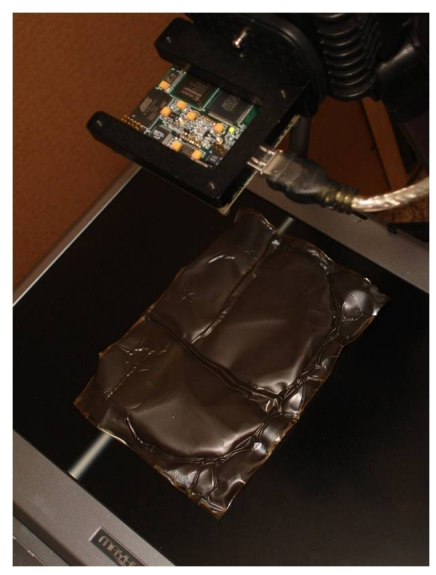

Figure 5: The scanner

The next pass of the stripes are captured with the document in the scene as shown in Fig. 5. The observed Gaussian intensity values for each camera pixel give $x_{0}^{\prime}, y_{0}^{\prime}, \sigma_{x}^{\prime}, \sigma_{y}^{\prime}$ as the estimates for the optically distorted Gaussian parameters.

For each pixel, we normalize the intensity values using the maximum observed intensity, $I_{\max }$ and $I_{\max }^{\prime}$, as the Gaussian stripe sweeps across the display. This scale factor, $\alpha=\frac{1}{I_{\max }}$ and $\alpha^{\prime}=\frac{1}{I_{\max }^{\prime}}$, is then saved as the visible intensity for that pixel. Then a non-linear fit is performed on the normalized intensity values to estimate the Gaussian parameters.

This gives us two 2D Gaussian functions for each pixel.

$$
G\left(x, y: x_{0}, y_{0}, \sigma_{x}, \sigma_{y}\right)=e^{\frac{\left(x-x_{0}\right)^{2}+\left(y-y_{0}\right)^{2}}{\left(\sigma_{x}+\sigma_{y}\right)^{2}}}
$$




$$
G\left(x, y: x_{0}^{\prime}, y_{0}^{\prime}, \sigma_{x}^{\prime}, \sigma_{y}^{\prime}\right)=e^{\frac{\left(x-x_{0}^{\prime}\right)^{2}+\left(y-y_{0}^{\prime}\right)^{2}}{\left(\sigma_{x}^{\prime}+\sigma_{y}^{\prime}\right)^{2}}}
$$

The difference between the Gaussian parameters in Eq. 5 and Eq. 6 gives an estimation of the optical changes due to the object in the scene which is discussed in Sec. 2.4 and 2.5.

\subsection{High-Dynamic Range Imaging}

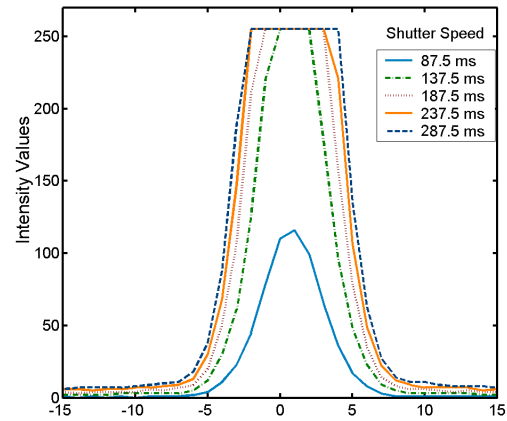

Figure 6: Shows the time-evolving intensity profile of 5 exposures for a single pixel using normal 8-bit images.

Quantization of intensity values during the imaging process greatly reduces the dynamic range in an image. This is shown in the longer exposures in Fig. 6. Consequently, this also reduces the accuracy of fitting intensity profiles to a Gaussian curve.

To compensate for this loss of data, High-Dynamic Range Imaging (HDRI) techniques have been developed [Debevec and Malik 1997] that use multiple images of the same scene under varying exposure rates. Then the response function for a scene is directly calculated using representative pixels under varying exposures. Once the response function is computed, the set of images can be combined into a floating point radiance map representative of the true radiance in the scene.

In this work, the developments in HDRI are extended to enhance the accuracy of the transmissive light acquisition. Instead of assuming one radiance value per pixel, radiance values are calculated for each position of the Gaussian stripe imaged by the pixel. Starting with the imaging of the same stripe with varying exposure rates, a pixel-wise radiance function can be estimated as shown in Fig. 7(a). The intensities of the pixel vary between the 8-bit intensity range of $0-255$ as the Gaussian stripe moves across the imaged sensor area. This over-constrains the linear least squares estimation of the response function:

$$
\begin{array}{r}
O=\sum_{x=1}^{X} \sum_{e=1}^{E}\left(w\left(I_{(x, e)}\right)\left(g\left(I_{(x, e)}\right)-\ln E_{x}-\ln \Delta t_{e}\right)\right)^{2} \\
+\lambda \sum_{z=Z_{\min }+1}^{Z_{\max }-1}\left(w(z) g^{\prime \prime}(z)\right)^{2}
\end{array}
$$

The weighting function $(w(z))$ was originally described as a simple hat function, however, in this work the compressed Gaussian peaks will play no role in the response curve computation. Therefore, the following weight function $w$ is used:

$$
w(z)= \begin{cases}z+1 & \text { if } z<=\frac{Z_{\max }-1}{2} \\ Z_{\max }-z+1 & \text { if } z>\frac{Z_{\max }}{2}\end{cases}
$$

The response function is estimated by choosing a single representative pixel that demonstrates a large dynamic range in the scene. Then the image response curve is defined by Eq. 9 .

$$
g\left(I_{(x, e)}\right)=\ln E_{x}+\ln \Delta t_{e}
$$

Fig. 7(b) shows a representative HDR Gaussian profile for a single pixel.

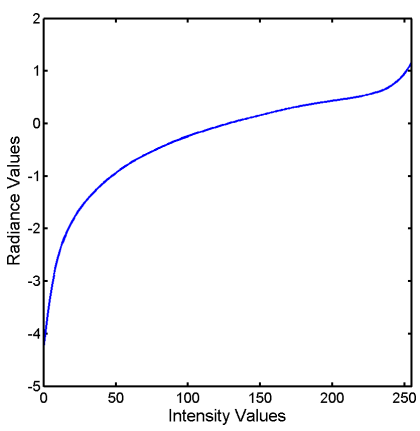

(a) The estimated response curve for the pixel in Fig. 6.

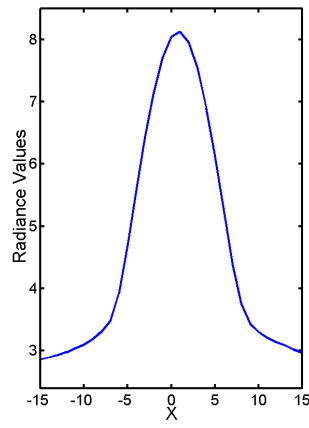
profile in radiance values.
Figure 7: The intermediate steps in calculating High Dynamic Range Imaging (HDRI).

\subsection{Acquiring Document Content}

The diffuse reflectance property of the ink is estimated in the system by change in Gaussian amplitude. Therefore, for single-layer documents the content can be approximated as $\frac{I_{\max }}{I_{\max }^{\prime}}$. However, for documents with more than one layer, attenuation may also include other factors that decrease transmission such as the channels in the photonegatives.

For these documents, the variance change $(\Delta \sigma)$ of the observed Gaussian stripes for each pixel, defined as the Eq. 10, provides a heavier weight on the content of the emulsion layer. When the variance is combined from each pixel into the entire image, the variance map is obtained by:

$$
\Delta \sigma=\operatorname{MAX}\left(\left(\sigma_{x}-\sigma_{x}^{\prime}\right), \gamma\left(\sigma_{y}-\sigma_{y}^{\prime}\right)\right)
$$

This work accommodates the non-square aspect ratio display devices by calculating difference in Dots-Per-Inch (DPI) for the $\mathrm{x}$ and y directions. This allows scaling of one axis by $\gamma$ to remove distortions due to aspect ratio and physical screen dimensions.

When $\sigma^{\prime}$ is much smaller than $\sigma$, it suggests that there is a much higher opacity in the content since the Gaussian width is reduced by the decreased transmission of light. Consequently when $\sigma^{\prime} \simeq$ $\sigma$, transmission is high which typically suggests that their is little content in the related pixel.

The variance map is converted using the following equation:

$$
I(u, v)=\left(V_{(u, v)}+t\right) s
$$

where $t$ is an intensity translation and $s$ is a scale factor. The values for $s$ and $t$ are determined empirically. 


\subsection{Distortion Shape Estimation}

The diffuse transmission of light can be used to directly estimate the surface orientation for each pixel observation on the document surface. Moreover, for non-planar documents relative variations in surface orientation provide a direct method to estimate local surface shape variations.

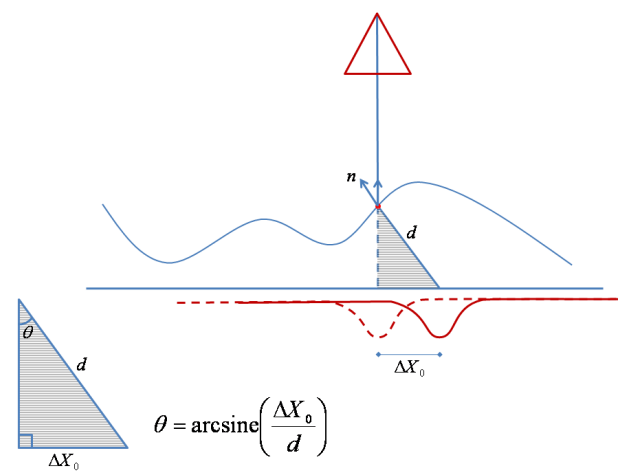

Figure 8: Diffuse Transmission of a back-lit light source

The change between the base position of the Gaussian stripe and the modified position provides a basic light-transport model for one or more layered documents. As the time-evolving Gaussian stripe moves across the display device, the observed transmitted intensities will also vary depending on the single-scatter diffusion given in Eq. 1 . The shift of the Gaussian peak, given by $\Delta X_{0}$ and defined by Eq. 12, gives a pixel-wise estimate for the change in light transmission through the document medium that may be used to estimate the surface orientation at the outermost layer of a document.

$$
\Delta X_{0}=\sqrt{\left(x_{0}^{2}-x_{0}^{\prime 2}\right)+\gamma\left(y_{0}^{2}-y_{0}^{\prime 2}\right.}
$$

This estimation for each pixel is combined across the entire image to obtain a distortion map $D(u, v)$.

When there is only one diffusely transmitting medium between the light source and the sensor, a direct estimation of the surface orientation for each pixel is possible. These pixel-wise orientations, given by $\left(\Delta x_{0}, \Delta y_{0}\right)$, are used to estimate the orientation of the surface for $x$ and $y$ directions.

$$
\Theta_{x}=\operatorname{arcsine}\left(\frac{\Delta x_{0}}{d_{x}}\right), \Theta_{y}=\operatorname{arcsine}\left(\frac{\Delta y_{0}}{d_{y}}\right)
$$

However, Eq. 13 have the unknown quantities $d_{x}$ and $d_{y}$ since the surface depth remains unknown as shown in Fig. 8. Therefore, for estimation purposes the mean values of both $\left(\Delta x_{0}\right.$ and $\left.\Delta y_{0}\right)$ are used for $d_{x}$ and $d_{y}$, so Eq. 13 becomes:

$$
\Theta_{x} \simeq \operatorname{arcsine}\left(\frac{\Delta x_{0}}{\left(\overline{\Delta x_{0}}\right)}\right), \Theta_{y} \simeq \operatorname{arcsine}\left(\frac{\Delta y_{0}}{\left(\overline{\Delta y_{0}}\right)}\right)
$$

It should be noted that the sign of these normal angles may be globally ambiguous. Similar to the bas-relief ambiguity in shape-fromshading [Belhumeur et al. 1999], the surface function may be the inverted version of the correct surface.

\subsubsection{Surface Reconstruction}

The surface gradient is defined as $\frac{\partial z}{\partial x} \simeq \frac{\Theta_{x}}{\sqrt{\Theta_{x}^{2}+\Theta_{y}^{2}+1}}$ in $x$ and $\frac{\partial z}{\partial y} \simeq \frac{\Theta_{y}}{\sqrt{\Theta_{y}^{2}+\Theta_{y}^{2}+1}}$ in $y$. With known surface gradients, an in- tegrable surface reconstruction can be calculated using a direct method [Frankot and Chellappa 1988].

\subsubsection{Perspective Projection Correction}

A global error is introduced into the normal map due to the prospective projection of the imaging system. As the distance from the camera's optical center increases, the angle of incidence on the surface also increases. This creates a systematic shift across the normal map that increases toward the edges of the image.

To compensate for this error, it is possible to work in the frequency domain where the error occurs. Since the error presents itself as very low-frequency noise, a Gaussian bandpass frequency filter is applied to the Fourier transform of surface gradient components in both the $X(u, v)$ and $Y(u, v)$ directions. The bandpass filter [Gonzalez and Woods 2001] uses two Gaussian distributions $G_{i}$, the starting frequency, and $G_{o}$, the closing frequency. Then the filters are combined to create a single bandpass filter $H=G_{i}-G_{o}$.

Once the filter is generated, the surface gradients may be filtered using $X^{\prime}(u, v)=X(u, v) H(u, v)$ and $Y^{\prime}(u, v)=Y(u, v) H(u, v)$. These processed values are then reduced of the error induced by perspective imaging. Therefore, the surface estimation more accurately portrays the actual document shape configuration. Fig. 9 shows the resultant surface as the low-frequency band-pass is decreased.

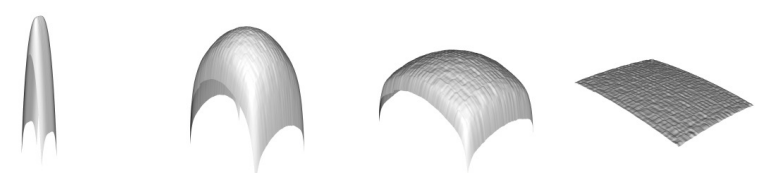

Figure 9: Estimated surface shape with decreasing low-frequency band-pass.

\section{Experimental Results}

To test the assumptions made in the previous section, a scanning system was built. The device consists of two pieces of hardware. A 1.6GHz Pentium M laptop with a 15"1024x768 LCD was used to run the scanning software and display the light patterns. Also driven by the laptop was a $640 \times 480$ 8-bit greyscale firewire camera. By keeping minimal hardware requirements, we hope to make the scanner available to the largest amount of users possible. The scan itself consists of displaying 650 vertical stripes and 400 vertical stripes for both the base and scanning steps. For each stripe position, 7 images are acquired with decreasing exposure speeds which requires 7350 images for each scan. The initial scans took roughly 0.5 second per image capture, so the entire scan took approximately 1 hour. Also, performing the non-linear Gaussian estimation for each pixel required 30 minutes.

To demonstrate the broad application of this scanner, we will show results of acquisition and restoration of both single and multiplelayer documents. For single-layer documents, pages with singlesided and duplex-sided text are scanned. Then to show the effectiveness of multi-layer document scanning, deteriorated photographic negatives are digitized and restored with the presented method.

\subsection{Single-layer documents}

The first result, Fig. 10, is a single-layer document with singlesided text. The front-illuminated image of the document is shown in 


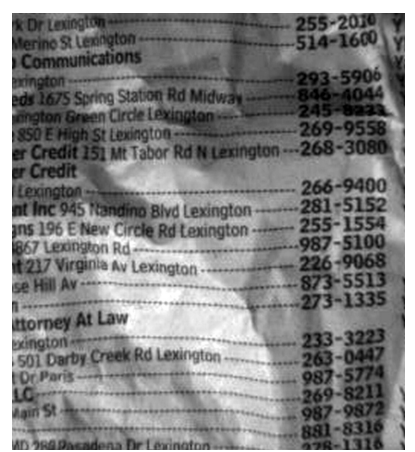

(a) The original image of a crumpled telephone book snippet.

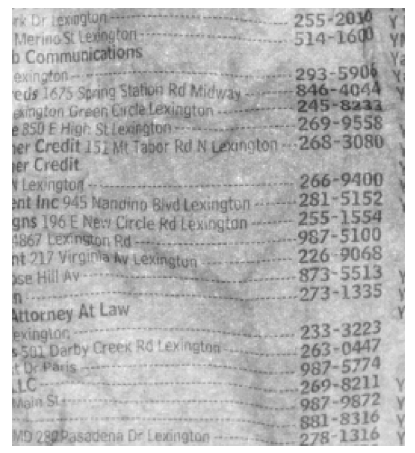

(c) The variance map.

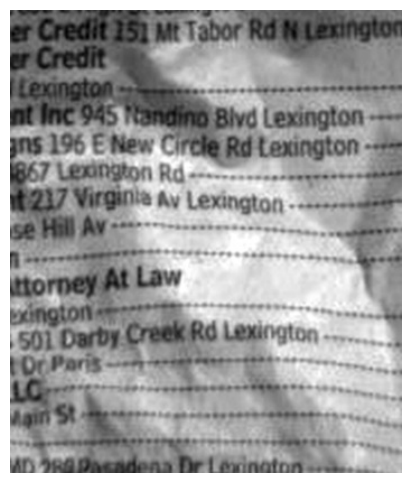

(e) A closeup of the original snippet

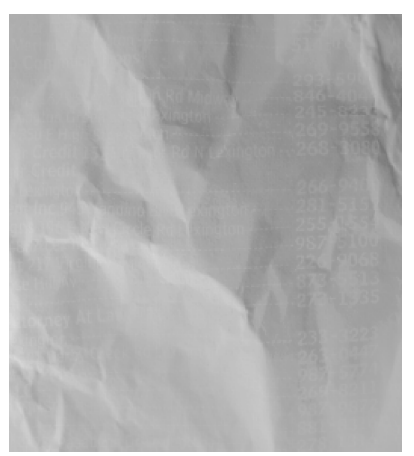

(b) The distortion map.

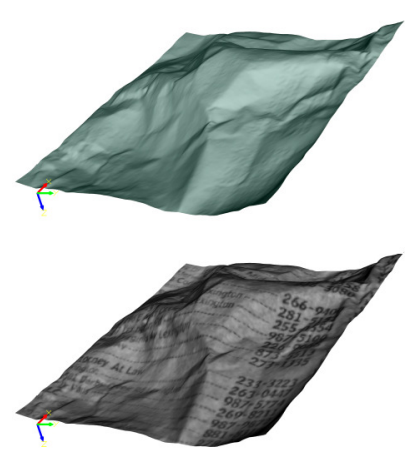

(d) Reconstructed 3D surface.

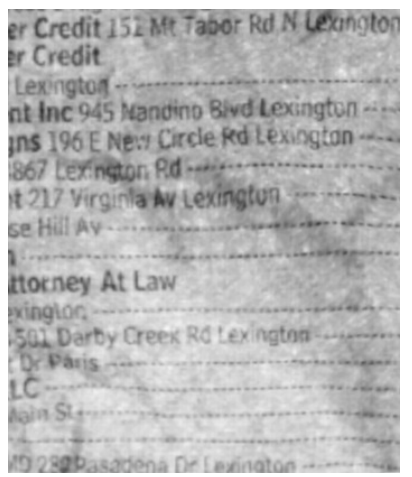

(f) The geometrically and photometrically corrected snippet.
Figure 10: An example phone directory with folds and wrinkles.

Fig. 10(a). Notice the deep folds that cause not only geometric distortion but also photometric distortion due to the non-uniform lighting. Once the surface is reconstructed, Fig. 10(b), and the intrinsic content obtained, Fig. 10(d), the completely restored document can be estimated. Using a physical simulation of a mass/spring system [Brown and Seales 2004], we simulate flattening the document back to its original planar form where Fig. 10(f) shows the final correction. The intrinsic content is calculated directly by the attenuation of the document substrate. Therefore, the composition of the document substrate does play a role in the restored image as can be seen by the speckled appearance of the paper.

\subsubsection{One-Pass Duplex-Sided Scanning}

The next result of the transmissive document scanner is onepass duplex-sided scanning. Single-paged documents with content printed on both sides can often be difficult to scan. Typical showthrough effects are shown in Figure 11(b). Most methods for scanning these documents focus on improving the accuracy for scanning a single side of the substrate. These techniques treat the intensity variations as artifacts that require removal in the final image. However, instead of treating show-through content as noise, the result presented here relies on the overall transmissivity of a document. As a result, the opacity of each layer can be segmented into a restored image of each side. Using the variance map, $\Delta \sigma$, for the document, the observed intensities may be subtracted from the variance map to remove content introduced from the front side of the page. The remaining content is from the rear-facing side of the document.

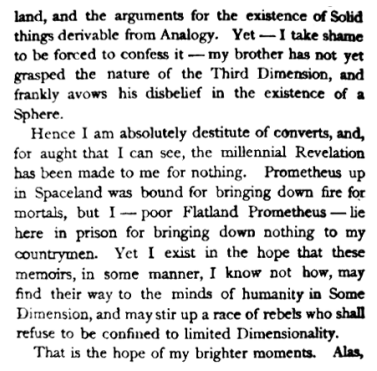

(a) Original image of the front illuminated document.

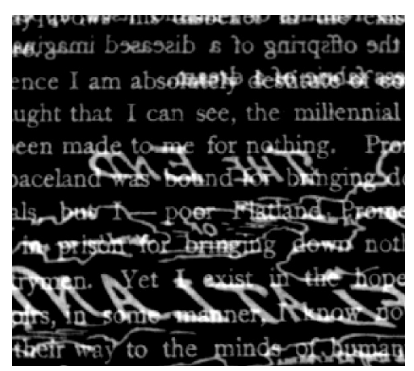

(c) The overall document attenuation.

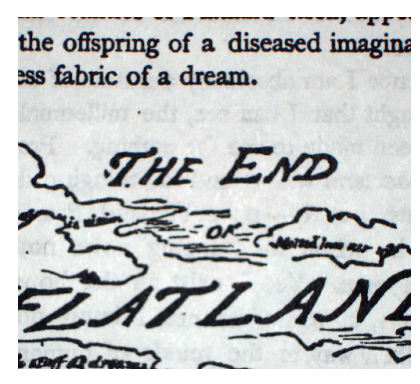

(b) A ground-truth photograph of the reverse side. Notice the showthrough effects from the opposite side.

the offspring of a diseased umagn ess fabone of a dream.

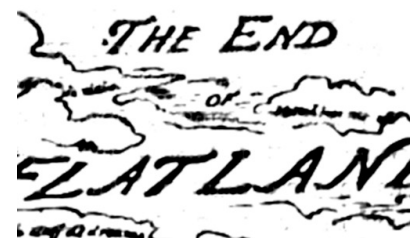

(d) Differentiated content from a scan of the opposite side of the document.
Figure 11: The process of extracting the reverse side content from a duplex-sided one-pass document scan.

Here we show how a book page with text printed on both sides can be efficiently scanned with a single-pass. By comparing the observed reflective image, Fig. 11(a), with the document attenuation, the reverse-side content, Fig. 11(c) is directly extracted as shown in Fig. 11(d). To compare the accuracy of this technique, a groundtruth image of the reverse side, Fig. 11(b), can be compared to the estimated result, Fig. 11(d).

\subsection{Multi-layer documents}

As discussed in the introduction, cellulose diacetate negative film collections make up a large number of archival photographs taken from 1925 to 1955 . The deteriorations from acetate shrinkage cause both photometric and geometric distortions. The photometric distortions occur when there is a separation between the base and emulsion layers of the document. The intrinsic intensities of the document can be directly estimated by $\Delta \sigma$.

The first result of restoring a photographic negative is performed on a recording of a monument. Fig. 1(a) shows how the separation between the layers creates channeling with non-uniform trans- 
mission of light when the negatives is imaged in the normal process. The photometrically corrected negative is shown in Fig. 1(c). The surface orientations are shown in Fig. 1(d). As can be seen by these images, the acquisition process effectively decouples the photographic content from the shape information while excluding attenuation effects caused by the layer separations.

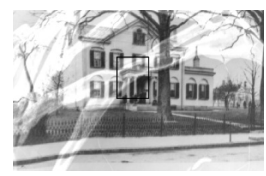

(a) The original deteriorated film negative.

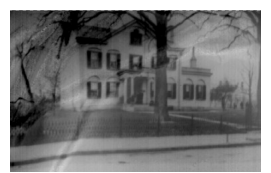

(b) The variance.

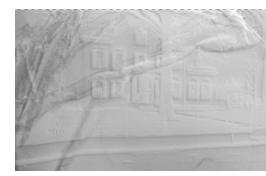

(c) The distortion map

Figure 12: An architectural photographic record from Lexington, Kentucky USA.

The next example is an architectural recording of a home. Fig. 12(a) shows the positive image of the photograph with obvious distortions in photometry and geometry. The photometrically corrected version of the negative is shown in Fig. 12(b) and the surface orientations are shown in Fig. 12(c).

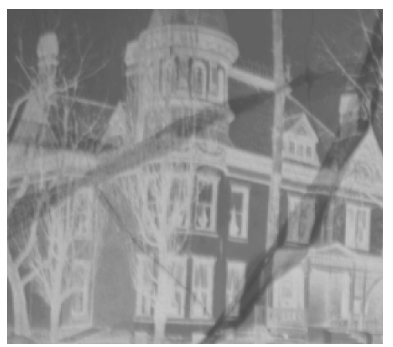

(a) Original negative image.

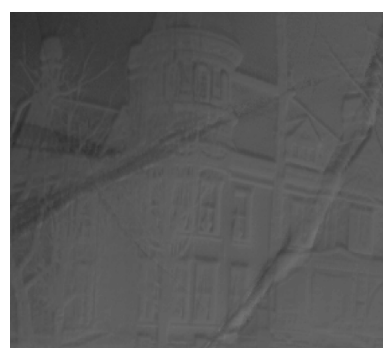

(c) The distortion map.

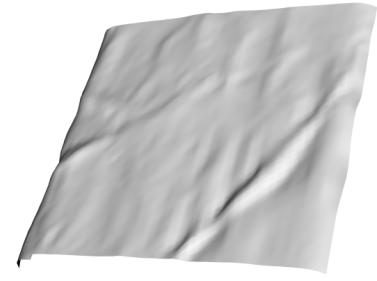

(e) Estimated surface for Fig. 13

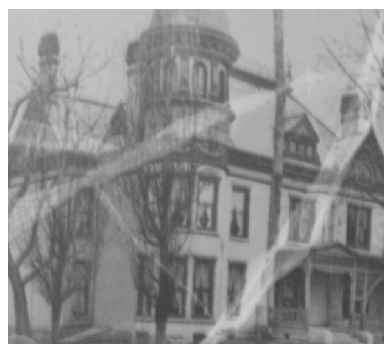

(b) Original positive image.

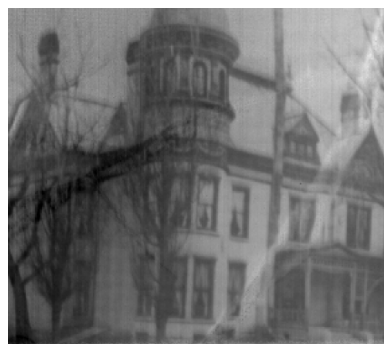

(d) The variance map.

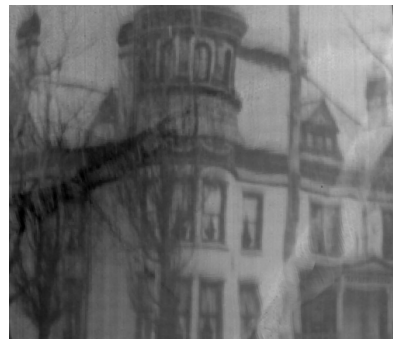

(f) The negative with both photometric and geometric error corrected.

Figure 13: Another architectural photographic record from Lexington, Kentucky USA.

The third example shows another architectural recording. Again, this negative suffers from the same severe deterioration that is com- mon in acetate film. Fig. 13(a) shows the negative acquired with a standard scanning process. The shape information is shown in Fig. 13(c) and the content is shown in Fig. 13(d). Fig. 13(e) shows

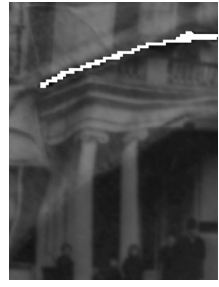

(a) A close-up (b) The corrected of the negative in result.

Fig. 12(a)
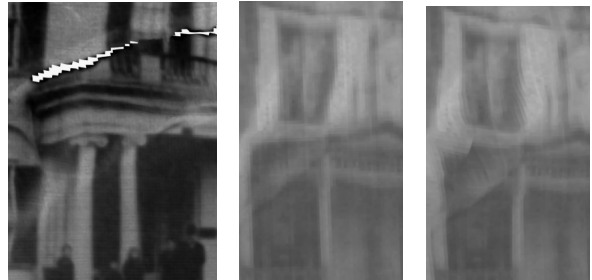

(c) A close-up (d) The flatof the negative tened result. in Fig. 13(d)
Figure 14: The geometric correction of negative from Figure 13.

the estimated surface. This geometry is then used for virtual flattening to correct dimensional warping with the result shown in Fig. 13(f).

Fig. 14(a) shows a closeup of a warped area of the negative from Fig. 12. In Fig. 14(a), a crack in the emulsion layer is marked in solid white. This area contains some information loss where the material has chipped away, but much of the content remains. It can be seen through the geometric flattening process shown in Fig. 14(b) that both sides of the crack are brought back together during restoration. Also, a close-up of Fig. 13 shows the resultant geometrically flattened negative in Fig. 14(d) with a side-by-side comparison on the unflattened photo (Fig. 14(c)).

\section{Future Work}

This work will be extended by developing a comprehensive groundtruth analysis suite to provide a metric for the overall restoration accuracy of the system. Also, future work will use more complex display patterns to greatly decrease the acquisition time. Moreover, increasing both the display and imaging resolution will achieve higher accuracy results.

\section{Conclusion}

In this work, we have demonstrated a cost-effective and fully automatic acquisition system that acquires shape and content information separately for single and multi-layer documents. Using singlescatter diffuse transmission as the basis for the document scanning system, successful results are shown. Single-layer documents with single-side and duplex-sided text are easily scanned with the system. Moreover, complex documents such as deteriorated multilayer photonegatives can be scanned and restored virtually. This presents the first known virtual restoration method for safety negatives.

\section{References}

Ardizzone, E., Dindo, H., Maniscalco, U., And MaZZOLA, G. 2006. Damages of digitized historical images as objects for content based applications. In Proceedings of the 14th European Signal Processing Conference, EURASIP.

Belhumeur, P. N., Kriegman, D. J., And Yuille, A. L. 1999. The bas-relief ambiguity. Int. J. Comput. Vision 35, 1, 33-44. 
BEn-EzRA, M., AND NAYAR, S. 2003. What does motion reveal about transparency? In Proceedings of the 9th IEEE International Conference on Computer Vision, vol. 2, 1025-1032.

Bertalmio, M., Sapiro, G., Caselles, V., And Ballester, C. 2000. Image inpainting. In Siggraph 2000, Computer Graphics Proceedings, ACM Press / ACM SIGGRAPH / Addison Wesley Longman, K. Akeley, Ed., 417-424.

Bertalmio, M., Vese, L., And Osher, G. S. S. 2003. Simultaneous structure and texture image inpainting. In Computer Vision and Pattern Recognition, IEEE Computer Society, vol. 2, 707-12.

Brown, M., AND SEALES, W. B. 2004. Image restoration of arbitrarily warped documents. IEEE Transactions on Pattern Analysis and Machine Intelligence 26, 10 (October), 1295-1306.

Brown, M., AND TsOI, Y. 2006. Geometric and shading correction for images of printed materials using boundary. IEEE Trans. Image Proc. 15, 6 (June), 1544-1554.

Bruni, V., Crawford, A., Kokaram, A. C., and VituLANO, D. 2007. Digital removal of blotches with variable semitransparency using visibility laws. In BVAI, 254-263.

CAO, H., Ding, X., And LiU, C. 2003. A cylindrical surface model to rectify the bound document image. In Proceedings of the 9th IEEE International Conference on Computer Vision, 228-233.

Chandrasekhar, S. 1960. Radiative transfer. Dover Publications. Unabridged and slightly revised verson of the work first published in 1950 .

ChICAgo Albumen 2008. http://www.albumenworks.com/detacetatefilms.html.

Chuang, Y.-Y., Zongker, D. E., Hindorff, J., Curless, B., SAlesin, D. H., AND Szeliski, R. 2000. Environment matting extensions: towards higher accuracy and real-time capture. In Proceedings of the 27th annual conference on Computer graphics and interactive techniques, ACM Press/AddisonWesley Publishing Co., New York, NY, USA, 121-130.

Criminisi, A., PÉREZ, P., AND ToyAmA, K. 2004. Region filling and object removal by exemplar-based image inpainting. IEEE Transactions on Image Processing 13, 9, 1200-1212.

DebeVec, P. E., AND MALIK, J. 1997. Recovering high dynamic range radiance maps from photographs. In Proceedings of the 24 th annual conference on Computer graphics and interactive techniques.

Frankot, R. T., AND Chellappa, R. 1988. A method for enforcing integrability in shape from shading algorithms. IEEE Trans. Pattern Anal. Mach. Intell. 10, 4, 439-451.

Frisvad, J. R., Christensen, N. J., ANd Falster, P. 2005. Efficient light scattering through thin semi-transparent objects. In Proceedings of the 3rd international conference on Computer graphics and interactive techniques in Australasia and South East Asia, ACM, New York, NY, USA, 135-138.

Ganapol, B., Johnson, L., Hammer, P., Hlavka, C., And Peterson, D. 1998. Leafmod: A new within-leaf radiative transfer model. Remote Sensing of Environment 63, 182-193.

Gonzalez, R. C., And Woods, R. E. 2001. Digital Image Processing. Addison-Wesley Longman Publishing Co., Inc., Boston, MA, USA.
Govaerts, Y. M., Jacquemoud, S., Verstraete, M. M., AND USTIN, S. L. 1996. Three-dimensional radiation transfer modeling in a dicotyledon leaf. Applied Optics 35 (November), 6585-6598.

HoRn, B. 1970. Shape from shading: a method for obtaining the shape of a smooth opaque object from one view. MIT Project Mac Technical Report TR-79.

Horvath, D. G. 1987. The Acetate Negative Survey: Final Report. The University of Louisville, Ekstrom Library, Photographic Archives, Louisville, KY 40292, February.

Landon, G. V., Lin, Y., And Seales, W. B. 2007. Towards automatic photometric correction of casually illuminated documents. In Computer Vision and Pattern Recognition, 2007. CVPR'07. IEEE Conference on.

MorRis, N. J. W., AND Kutulakos, K. N. 2007. Reconstructing the surface of inhomogeneous transparent scenes by scatter trace photography. In Proceedings of the 11th IEEE International Conference on Computer Vision.

Roth, S., AND BLACK, M. J. 2005. Fields of experts: A framework for learning image priors. In Proceedings of the IEEE Computer Society Conference on Computer Vision and Pattern Recognition, IEEE Computer Society, Washington, DC, USA, 860-867.

SEALES, W. B., AND LIN, Y. 2004. Digital restoration using volumetric scanning. In Proceedings of the 4th ACM/IEEE-CS joint conference on Digital libraries, ACM, New York, NY, USA, 117-124.

Stanco, F., RAmponi, G., And DE Polo, A. 2003. Towards the automated restoration of old photographic prints: a survey. The IEEE Region 8 EUROCON 20032 (September), 370-374.

Stanco, F., Tenze, L., Ramponi, G., And De Polo, A. 2004. Virtual restoration of fragmented glass plate photographs. Proceedings of the 12th IEEE Mediterranean Electrotechnical Conference 1 (May), 243-246.

Sun, M., Yang, R., Lin, Y., Landon, G. V., Seales, W. B., AND BROWN, M. S. 2005. Geometric and photometric restoration of distorted documents. In Proceedings of the 10th IEEE International Conference on Computer Vision, vol. 2, 1117- 1123.

SzEliski, R., AVIDAn, S., AND ANANDAN, P. 2000. Layer extraction from multiple images containing reflections and transparency. In Proceedings of IEEE Conference on Computer Vision and Pattern Recognition, vol. 1, 246-253.

Wang, L., Wang, W., Dorsey, J., YAng, X., Guo, B., And SHUM, H.-Y. 2006. Real-time rendering of plant leaves. In SIGGRAPH '06: ACM SIGGRAPH 2006 Courses, ACM Press, New York, NY, USA, 5.

ZhANG, L., Zhang, Y., AND TAN, C. 2007. An improved physically-based method for geometric restoration of distorted document images. IEEE Transactions on Pattern Analysis and Machine Intelligence (November).

Zongker, D. E., Werner, D. M., Curless, B., AND SALESIN, D. H. 1999. Environment matting and compositing. In Proceedings of the 26th annual conference on Computer graphics and interactive techniques, ACM Press/AddisonWesley Publishing Co., New York, NY, USA, 205-214. 